\title{
ABDELKADER ELQORTOBI Inf-convolution quasi-convexe des fonctionnelles positives
}

Revue française d'automatique, d'informatique et de recherche opérationnelle. Recherche opérationnelle, tome 26, nº 4 (1992), p. 301-311.

<http://www.numdam.org/item?id=RO_1992_26_4_301_0>

(C) AFCET, 1992, tous droits réservés.

L'accès aux archives de la revue « Revue française d'automatique, d'informatique et de recherche opérationnelle. Recherche opérationnelle » implique l'accord avec les conditions générales d'utilisation (http://www.numdam.org/ legal.php). Toute utilisation commerciale ou impression systématique est constitutive d'une infraction pénale. Toute copie ou impression de ce fichier doit contenir la présente mention de copyright.

\section{Numdam}

Article numérisé dans le cadre du programme

Numérisation de documents anciens mathématiques

http://www.numdam.org/ 
Recherche opérationnelle/Operations Research

(vol. 26, n 4,1992, p. 301 à 311)

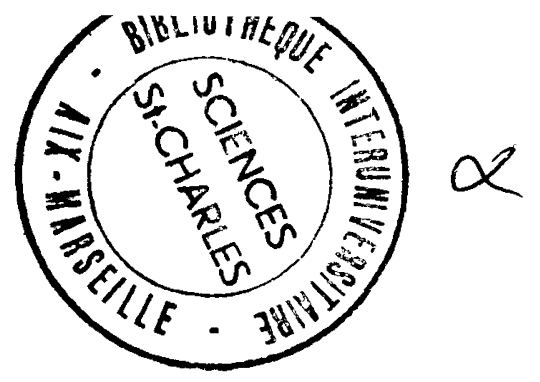

\title{
INF-CONVOLUTION QUASI-CONVEXE DES FONCTIONNELLES POSITIVES ( $\left.{ }^{*}\right)$
}

\author{
par Abdelkader ELQORTOBI ( $\left.{ }^{1}\right)$ \\ Communiqué par J.-P. CrouzelX
}

Résumé. - L'inf-convolution convexe joue un rôle prépondérant en analyse convexe. Elle est étroitement liée à la conjuguée convexe d'une fonctionnelle. C'est pourquoi on va utiliser la définition de l'inf-convolution quasi-convexe pour définir notre polaire qui se trouve avoir un rapport avec celles définies par Crouzeix d'une part et Greenberg \& Pierskalla $(G \& P)$ d'autre part. On définira son quasi-tangentiel et on terminera par un exemple $\cdot$ la «surrogate dualité».

Mots clés : Optimisation; quasi-convexe; quasi-tangentiel; inf-convolution.

Abstract. - The infimal convolution has an important role in convex analysis. It is closely related to a polar of a convex function. We use the definition of a quasiconvex infimal convolution to define our polar which is related to the polars defined respectively by Crouzeix and Greenberg \& Pierskalla $(G \& P)$. In this work, we shall define its quasi-tangential and we shall end by an example: the "surrogate duality".

Keywords : Optimization; quasiconvexity; quasi-tangential; infimal convolution.

\section{INTRODUCTION}

Dans tout ce qui suit, $X$ et $X^{\prime}$ désignent deux espaces vectoriels topologiques localement convexes mis en dualité séparante par la forme bilinéaire notée $\langle\rangle,, \mathbb{P}=\{f: X \rightarrow[0,+\infty]\}$ et $\mathbb{F}$ sera l'ensemble $\bar{R}^{X}=\{f: X \rightarrow[-\infty,+\infty]\}$. On prendra soin de prolonger l'addition des réels à $\widetilde{R}=[-\infty,+\infty]$ de la manière suivante: $a+(+\infty)=+\infty$ pour tout $a$ appartenant à $[-\infty,+\infty]$ (cf. [7]). Rappelons les principales définitions.

(*) Reçu en mai 1991.

( $\left.{ }^{1}\right)$ Département de mathématiques et d'informatique, Université de Sherbrooke, Sherbrooke (Québec), Canada. 
L'épigraphe strict de $f$ noté est $(f)$ est l'ensemble

$$
\text { est }(f)=\{(x, \lambda) \in X \times R: f(x)<\lambda\}
$$

et les tranches ou sections de $f$ sont les ensembles suivants

$$
\mathrm{T}(f, \lambda)=\{x \in X: f(x) \leqq \lambda\} \quad \text { et } \quad t(f, \lambda)=\{x \in X: f(x)<\lambda\} \text { où } \lambda \text { est réel. }
$$

La conjuguée convexe de $f$ est définie par

$$
f^{*}\left(x^{\prime}\right)=\operatorname{Sup}\left\{\left\langle x, x^{\prime}\right\rangle-f(x): x \in X\right\}
$$

Enfin on dira que $f$ est propre si le domaine effectif de $f$ est non vide et si $f$ est finie sur ce domaine. L'inf-convolution convexe de deux fonctionnelles $f$ et $g$ introduite par Moreau ( $c f$. [7]) est :

$$
f \Delta g(z)=\operatorname{Inf}\{f(x)+g(z-x): x \in X\}
$$

La loi $\Delta$ vérifie l'égalité : est $(f \Delta g)=$ est $(f)+$ est $(g)$

Remplaçons $g$ par un élément $x^{\prime}$ de $X^{\prime}$. On obtient

$$
\begin{aligned}
f \Delta x^{\prime}(z)=\operatorname{Inf}\left\{f(x)+\left\langle z-x, x^{\prime}\right\rangle: x \in X\right\} \\
\quad=-\operatorname{Sup}\left\{\left\langle x-z, x^{\prime}\right\rangle-f(x): x \in X\right\}=\left\langle z, x^{\prime}\right\rangle-f^{*}\left(x^{\prime}\right)
\end{aligned}
$$

Si on pose $f_{z}^{*}\left(x^{\prime}\right)=\left\langle z, x^{\prime}\right\rangle-f^{*}\left(x^{\prime}\right)$ pour $z$ fixé appartenant à $X$, alors on déduit facilement les propriétés suivantes:

(i) $f_{z}^{*}(0)=\operatorname{Inf} f$ et $-f_{0}^{*}=f^{*}$

(ii) $\operatorname{Sup}\left\{f_{z}^{*}\left(x^{\prime}\right): x^{\prime} \in X^{\prime}\right\}=f^{* *}(z)=\operatorname{Sup}\left\{f \Delta x^{\prime}(z): x^{\prime} \in X^{\prime}\right\}$

(iii) $-f_{z}^{*}$ est convexe (cx) et semi-continue inférieurement (sci).

On va utiliser cette approche pour définir notre polaire en utilisant l'infconvolution quasi-convexe (qcx). Cette contribution originale s'ajoute à celles déjà existantes. On rappelle que Crouzeix et Greenberg \& Pierskalla utilisent, chacun à leur manière, une décomposition de $f^{*}$ pour définir leur conjuguée (cf. [3], [4]). Dans [1], les auteurs appliquent deux polarités particulières aux tranches de fonctions pour définir leur conjuguée. Passy \& Prisman utilisent les fonctions homogènes et la notion de "evenly» convexe pour définir une conjugaison symétrique. Dans [12], Volle a généralisé toutes ces notions. Pour d'autres approches, on pourra consulter par exemple [6], [9], [11], etc.

Dans tout ce qui suit, on utilisera la notation suivante: $\operatorname{Max}(a, b)=a \vee b$ 


\section{PROPRIETES DE L'INF-CONVOLUTION QUASI-CONVEXE}

L'inf-convolution quasi-convexe introduite par Rockafellar (cf. [10]) est définie comme suit:

$$
f \nabla g(z)=\operatorname{Inf}\{f(x) \vee g(z-x): x \in X\}
$$

On a l'importante égalité :

$$
t(f \nabla g, \lambda)=t(f, \lambda)+t(g, \lambda) \quad \text { pour tout } \lambda \text { réel }
$$

Remarquons que si on remplace la fonction $g$ par la fonction nulle, on obtient

$$
f \nabla 0(z)=\operatorname{Inf}\{f(x) \vee 0: x \in X\}
$$

ce qui ne correspond pas en général à Inf $f$. Pour pallier à cet inconvénient, il suffira de considérer des fonctionnelles positives.

Avant de définir notre fonction polaire, étudions quelques propriétés de l'opération $\nabla$ et rappelons certaines définitions relatives à la quasi-convexité.

$f$ est quasi-convexe (qcx) si $f(\lambda u+(1-\lambda) v) \leqq f(u) \vee f(v)$ pour tout $(u, v, \lambda)$ appartenant à $X \times X \times[0,1], f$ est quasi-concave (qcv) si $-f$ est quasi-convexe.

On a aussi le résultat suivant: $f$ est $\mathrm{qcx} \Leftrightarrow$ les ensembles $T(f, \lambda)$ ou $t(f, \lambda)$ sont convexes pour tout $\lambda$ réel.

L'opération $\nabla$ conserve la convexité et la quasi-convexité des fonctionnelles (cf. [10]) autrement dit

$$
f \text { et } g \text { convexe } \Rightarrow f \nabla g \text { convexe }
$$

$f$ et $g$ quasi-convexe $\Rightarrow f \nabla g$ quasi-convexe

THÉORÈme 1 : Soient $A$ et $B$ des ensembles non vides, $f$ et $g$ des fonctionnelles appartenant à $\bar{R}^{X}$. Désignons par $\delta_{A}$ la fonction indicatrice de l'ensemble $A$.

(1) $\nabla$ est commutative et associative.

(2) (i) $\operatorname{dom} f \nabla g=\operatorname{dom} f+\operatorname{dom} g$; (ii) $\delta_{A} \nabla \delta_{B}=\delta_{A+B}$.

(3) $f \nabla \delta_{\{x\}}(z)=f(z-x) \vee 0$. Si f est positive alors $f \nabla \delta_{\{x\}}(z)=f(z-x)$. On remarque alors que pour tout $\mu$ positive $t\left(f \nabla \delta_{\{z\}}, \mu\right)=t(f, \mu)+\{z\}$.

(4) $f \leqq g \Rightarrow f \nabla h \leqq g \nabla h$ pour toute fonctionnelle $h$.

(5) $\operatorname{Inf}\left\{f_{i} \nabla g_{j}:(i, j) \in I \times J\right\}=\operatorname{Inf}\left\{f_{i}: i \in I\right\} \nabla \operatorname{Inf}\left\{g_{j}: j \in J\right\}$ pour toutes familles de fonctions $\left(f_{i}\right)$ et $\left(g_{j}\right)$.

(6) Soit $N$ une norme sur $X$. Si d $(z, A)$ désigne la distance de $z$ à $A$, alors:

$$
N \nabla \delta_{A}(z)=\operatorname{Inf}\{N(z-x): x \in A\}=d(z, A) .
$$


(7) Soit $f$ positive. $f \nabla \delta_{-A}(z)=\operatorname{Inf}\{f(x): x \in A+z\}$ et en particulier $f \nabla \delta_{-A}(0)=\operatorname{Inf}\{f(x): x \in A\}$.

Preuve: Immédiat.@

DÉfINITION 1 : Soit $f$ appartenant à $\mathbb{F}$. On dit que $f$ est inf-compacte si pour tout $\mu$ réel l'ensemble $T(f, \mu)$ est compact.

THÉORÈme 2 : Soient $f$ et $g$ deux éléments propres de $\mathbb{F}$ telles que $f$ soit infcompacte et $g$ soit sci. Alors $f \nabla g$ est sci, bornée inférieurement et $\nabla$ est exacte.

Preuve. - Par construction de la fonction $f \nabla g$, on a :

$$
T(f \nabla g, \mu)=\bigcap\{T(f, \beta)+T(g, \beta): \beta>\mu\}
$$

Comme $T(f, \beta)$ est compact et que $T(g, \beta)$ est fermé, il s'ensuit que $T(f \nabla g, \mu)$ est fermé, par conséquent $f \nabla g$ est sci.

Comme Inf $f \leqq f \nabla g(z)$ pour tout $z$ appartenant à $X$ et que $f$ est infcompacte (donc minorée), on déduit que $f \nabla g$ est bornée inférieurement.

La fonction $k: x \rightarrow f(x) \vee g(z-x)$ est sci comme maximum de deux fonctions sci. En plus le fermé $T(k, \beta)$ est inclus dans le compact $T(f, \beta)$. Par conséquent la fonction $k$ est inf-compacte et donc minorée. Elle admet donc un minimum sur $X$ non égal à $-\infty$. L'inf-convolution est donc exacte c'està-dire $f \nabla g(z)=\operatorname{Min}\{f(x) \vee g(z-x): x \in X\}$ pour tout $z$ appartenant à $X$ (autrement dit il existe $u$ et $v$ appartenant à $X$ tels que $u+v=z$ et $f \nabla g(z)=f(u) \vee g(v))$. @

Remarque 1: Si $f$ et $g$ sont deux fonctionnelles propres et inf-compactes de $\mathbb{F}$, alors $f \nabla g$ est également propre et inf-compacte. Ceci est une conséquence directe de l'expression de $T(f \nabla g, \mu)$ vue précédemment.

\section{POLAIRE D'UNE FONCTIONNELLE}

DÉfINITION 2 : Soit $f$ une fonction positive. La fonction polaire de $f$ en un point $z$ appartenant à $X$ est définie par:

$F_{z}\left(x^{\prime}\right)=\operatorname{Inf}\left\{f(x) \vee\left\langle z-x, x^{\prime}\right\rangle: x \in X\right\}=f \nabla x^{\prime}(z)$ pour tout $x^{\prime}$ appartenant à $X^{\prime}$

On posera aussi:

$$
f^{E}(z)=\operatorname{Sup}\left\{F_{z}\left(x^{\prime}\right): x^{\prime} \in X^{\prime}\right\}=\operatorname{Sup}\left\{f \nabla x^{\prime}(z): x^{\prime} \in X^{\prime}\right\}
$$


Remarque 2:

(i) La fonction $F_{z}$ est obtenue de la définition de l'inf-convolution quasiconvexe et vérifie $F_{z}(0)=\operatorname{Inf} f$,

(ii) La fonction $F_{z}$ dépend de $z$; cependant on la notera souvent $F$ s'il n'y a pas d'ambiguité.

Rappelons l'expression des polaires $q^{C}$ et $q^{G}$ en un point $z$ définies respectivement par Crouzeix et G \& $P(c f$. [3], [4]):

$$
\begin{gathered}
q^{c}\left(z, x^{\prime}\right)=\operatorname{Inf}\left\{\lambda: \operatorname{Inf}\left\{\left\langle z-x, x^{\prime}\right\rangle: f(x) \leqq \lambda\right\} \leqq 0\right\} \\
q^{G}\left(z, x^{\prime}\right)=\operatorname{Inf}\left\{f(x):\left\langle z-x, x^{\prime}\right\rangle \leqq 0\right\}
\end{gathered}
$$

Étudions maintenant les propriétés des fonctions $F_{z}$ et $f^{E}$.

THÉORÈME 3 : Soient $f$ et $g$ des fonctionnelles positives.

(1) $f \leqq g \Rightarrow F \leqq G \Rightarrow f^{E} \leqq g^{E}$

(2) Si on pose $h=\mu$ f pour tout $\mu>0$, alors $H\left(x^{\prime}\right)=\mu F\left(x^{\prime} / \mu\right)$

(3) (i) $F_{z}\left(x^{\prime}\right) \leqq f(z)$ pour tout $\left(z, x^{\prime}\right)$ appartenant à $X \times X^{\prime}$; (ii) $f^{E} \leqq f$

(4) (i) $f=\operatorname{Sup} f_{i} \Rightarrow \operatorname{Sup} F_{i} \leqq F$ pour toute famille de fonctions positives $f_{i}$;

(ii) $f=\operatorname{Inf} f_{i} \Rightarrow \operatorname{Inf} F_{i}=F$ pour toute famille de fonctions positives $f_{i}$

(5) $t\left(F_{z}, \mu\right)=\bigcup_{x \in t(f, \mu)}\left\{x^{\prime} \in X^{\prime}:\left\langle z-x, x^{\prime}\right\rangle<\mu\right\}$

(6) $T\left(f \nabla x^{\prime}, \mu\right)=\bigcap_{\lambda>\mu}\left\{z \in X:\left\langle z, x^{\prime}\right\rangle \leqq \lambda+\delta_{t(f, \lambda)}^{*}\left(x^{\prime}\right)\right\}$

(7) $T\left(f^{E}, \mu\right)=\bigcap_{x^{\prime} \in X^{\prime}} \bigcap_{\lambda>\mu} \bigcup_{\left\langle x, x^{\prime}\right\rangle<\lambda} t\left(f \nabla \delta_{\{z\}}, \lambda\right)$

Preuve: (1), (2), (3), (4) et (5) sont immédiats.

(6) $\left\{z:\left\langle z, x^{\prime}\right\rangle \leqq \lambda+\delta_{t(f, \lambda)}^{*}\left(x^{\prime}\right)\right\}$

$$
\begin{aligned}
= & \cap\left\{\left\{z:\left\langle z, x^{\prime}\right\rangle\left\langle\lambda+\varepsilon+\delta_{t(f, \lambda)}^{*}\left(x^{\prime}\right)\right\}: \varepsilon>0\right\}\right. \\
& =\cap\left\{\left\{z: \operatorname{Inf}\left\{f(x)+\varepsilon \vee\left\langle z-x, x^{\prime}\right\rangle: x \in X\right\}<\lambda+\varepsilon\right\}: \varepsilon>0\right\}
\end{aligned}
$$

Par conséquent

$$
\begin{aligned}
\bigcap_{\lambda>\mu}\{z \in X: & \left.\left\langle z, x^{\prime}\right\rangle \leqq \lambda+\delta_{t(f, \lambda)}^{*}\left(x^{\prime}\right)\right\} \\
& =\bigcap_{\lambda>\mu} \bigcap_{\varepsilon>0}\left\{z: \operatorname{Inf}\left\{f(x)+\varepsilon \vee\left\langle z-x, x^{\prime}\right\rangle: x \in X\right\}<\lambda+\varepsilon\right\} \\
& =\bigcap_{\varepsilon>0} \bigcap_{\lambda>\mu}\left\{z: \operatorname{Inf}\left\{f(x)+\varepsilon \vee\left\langle z-x, x^{\prime}\right\rangle: x \in X\right\}<\lambda+\varepsilon\right\} \\
& =\cap\left\{\left\{z: \operatorname{Inf}\left\{f(x)+\varepsilon \vee\left\langle z-x, x^{\prime}\right\rangle: x \in X\right\} \leqq \mu+\varepsilon\right\}: \varepsilon>0\right\}
\end{aligned}
$$




$$
\begin{array}{r}
=\left\{z \in X: \operatorname{Inf}\left\{f(x) \vee\left\langle z-x, x^{\prime}\right\rangle: x \in X\right\} \leqq \mu\right\}=T\left(f \nabla x^{\prime}, \mu\right) \\
T\left(f^{E}, \mu\right)=\bigcap_{\lambda>\mu}\left\{z \in X: f^{E}(z)<\lambda\right\}=\bigcap_{\lambda>\mu} \bigcap_{x^{\prime} \in X^{\prime}}\left\{z \in X: F_{z}\left(x^{\prime}\right)<\lambda\right\}
\end{array}
$$

Or pour $x^{\prime}$ fixé,

$$
\begin{aligned}
& F_{z}\left(x^{\prime}\right)<\lambda \Leftrightarrow \exists x \in X \quad \text { tel que } f(x)<\lambda \text { et } \quad\left\langle z-x, x^{\prime}\right\rangle<\lambda \\
& \Leftrightarrow \exists x \in X \text { tel que } f(z-x)<\lambda \text { et }\left\langle x, x^{\prime}\right\rangle<\lambda \\
& \Leftrightarrow \exists x \in X \quad \text { tel que } z \in t(f, \lambda)+\{x\} \text { et }\left\langle x, x^{\prime}\right\rangle<\lambda
\end{aligned}
$$

Par suite

$$
\left\{z \in X: F_{z}\left(x^{\prime}\right)<\lambda\right\}=\bigcup_{\left\langle x, x^{\prime}\right\rangle<\lambda} t(f, \lambda)+\{x\}=\bigcup_{\left\langle x, x^{\prime}\right\rangle<\lambda} t\left(f \nabla \delta_{\{z\}}, \lambda\right) . @
$$

ThÉORÈme 4 : Soit $f \in \overleftarrow{R}^{X}$.

(i) $F$ est quasi-concave et semi-continue supérieurement (scs) par rapport à $x^{\prime}$.

(ii) $F$ est quasi-concave, quasi-convexe et semi-continue inférieurement (sci) par rapport $\grave{a} z$.

Preuve:

(i) Immédiat d'après Théorème 3,5 .

(ii) D'après Théorème $3,6, F$ est qcx et sci.

Pour prouver que $F$ est qcv et scs, il suffit de considérer la fonctionnelle

$$
\varphi_{x}\left(x^{\prime}, z\right)=f(x) \vee\left\langle z-x, x^{\prime}\right\rangle \text { pour } x \text { fixé dans } X
$$

qui, pour $x$ et $x^{\prime}$ fixés, est qcv et scs en $z$ d'où $F$ est qcv et scs comme infimum de fonctions qcv et scs. @

COROllaire $2: f^{E}$ est qcx et sci par rapport à $z$.

Preuve: $f^{E}$ est la borne supérieure d'une famille de fonctions qcx et sci. @

ThÉORÈme 5 : Soient $f$ qcx appartenant à $P$ et $z$ appartenant $\grave{a} X$. Si $f$ est sci en $z$ alors $f^{E}(z)=f(z)$.

Preuve: Il suffit de prouver que $f^{E}(z) \geqq f(z)$.

(a) Supposons que $f(z)=\operatorname{Inf} f$. En prenant $x^{\prime}=0$, on obtient:

$$
f^{E}(z) \geqq \operatorname{Inf}\{f(x) \vee\langle z-x, 0\rangle: x \in X\}=\operatorname{Inf} f=f(z) .
$$


(b) Supposons que $\operatorname{Inf} f<f(z)$. Soit $\lambda$ tel que $\operatorname{Inf} f<\lambda<f(z)$. Puisque $f$ est sci en $z$, il existe un voisinage convexe ouvert $V$ de $z$ tel que pour tout $v$ appartenant à $V, \lambda<f(v)$. Par suite $V \cap T(f, \lambda)=\varnothing$. D'après un théorème de séparation de Hahn-Banach ( $c f$. [5]), il existe un hyperplan fermé qui les sépare. Plus précisément,

$$
\exists x^{\prime} \neq 0 \text { tel que } \forall x \in T(f, \lambda), \lambda \leqq\left\langle z-x, x^{\prime}\right\rangle
$$

et

$$
\forall x \in V \quad\left\langle z-x, x^{\prime}\right\rangle \leqq \lambda \quad \text { car } \quad 0 \in V .
$$

Il s'ensuit que

$$
\lambda \leqq \operatorname{Inf}\left\{\operatorname{Max}\left(f(x),\left\langle z-x, x^{\prime}\right\rangle\right): x \in T(f, \lambda)\right\}
$$

et

$$
\lambda \leqq \operatorname{Inf}\left\{\operatorname{Max}\left(f(x),\left\langle z-x, x^{\prime}\right\rangle\right): x \notin T(f, \lambda)\right\} .
$$

On obtient donc $\lambda \leqq F_{z}\left(x^{\prime}\right)$ et par conséquent $\lambda \leqq f^{E}(z)$ pour tout $\lambda$ vérifiant $\lambda<f(z)$ ce qui veut dire que $f(z) \leqq f^{E}(z)$. @

Corollaire 3 : Soit $f$ appartenant à P. Alors $f^{E E}=f^{E}$

Preuve: Il suffit de poser $g=f^{E}$ et d'employer le théorème précédent à $g$. @

Remarque 3: On vérifie aisément les inégalités suivantes ( $c f .[3])$ :

$$
f^{E}(z) \leqq f_{q i}(z)=\operatorname{Sup} q^{C}\left(z, x^{\prime}\right) \leqq f^{G G}(z)=\operatorname{Sup} q^{G}\left(z, x^{\prime}\right) \leqq f_{q}(z) \leqq f(z)
$$

où la fonctionnelle $f_{q i}$ (resp. $f_{q}$ ) est la plus grande minorante qcx et sci (resp. qcx) de $f$.

Corollaire 4 : Soit $f$ appartenant à $P . f^{E}$ est la plus grande minorante $q c x$ et sci de $f$, autrement dit elle coïncide avec $f_{q i}$.

Preuve: Soit $g$ une minorante qcx et sci de $f$. On déduit que $g^{E} \leqq f^{E}$. D'après le théorème précédent, $g^{E}=g$ d'où $g \leqq f^{E}$. @

Exemple: Prenons l'exemple cité dans [4]: soit $\lambda \in[0,1]$ et $f(x)=0$ si $x<0$, $\lambda$ si $x=0$ et 1 sinon. Suivant la valeur $\lambda$, on peut rendre $f$ sci ou non.

(a) Pour $0<z, F_{z}\left(x^{\prime}\right)=1$ si $z x^{\prime} \geqq 1, z x^{\prime}$ si $0<z x^{\prime}<1$ et 0 sinon

(b) Pour $0 \geqq z, F_{z}\left(x^{\prime}\right)=0$.

On obtient alors: $f^{E}(z)=0$ si $z \leqq 0$ et 1 sinon. 


\section{QUASI-TANGENTIEL}

Crouzeix définie le tangentiel de $f$ en $z$ comme étant le sous-ensemble $T f(z)$ de $X^{r}$ tel que :

$$
x^{\prime} \in T f(z) \Leftrightarrow f(z)=q^{c}\left(z, x^{\prime}\right)
$$

Il est alors naturel de poser la définition suivante.

Définition 3 : Soit $f \in P$.

$x^{\prime}$ est un sous-gradient de $f$ en $z$ si et seulement si $f(z)=F_{z}\left(x^{\prime}\right)$.

Le quasi-tangentiel de $f$ en $z$, noté $Q f(z)$, est l'ensemble (éventuellement vide) des sous-gradients de $f$ en $z$. On a donc:

$$
x^{\prime} \in Q f(z) \Leftrightarrow f(z)=F_{z}\left(x^{\prime}\right)
$$

THÉORÈme 6 : Soient $f \in P$ et $z \in X$.

(1) $Q f(z)$ est un convexe fermé

(2) $0 \in Q f(z) \Leftrightarrow f(z)=\operatorname{Inf} f$

(3) $Q f(z) \neq \varnothing \Rightarrow f(z)=f^{E}(z)$

(4) $Q f(z) \subseteq T f(z)$.

Preuve: (1), (2) et (3) sont immédiats.

(4) Il suffit de prouver que $F_{z}\left(x^{\prime}\right) \leqq q^{c}\left(z, x^{\prime}\right)$.

Supposons le contraire, c'est-à-dire il existe $\left(z, x^{\prime}\right)$ appartenant à $X \times X^{\prime}$ tel que $F_{z}\left(x^{\prime}\right)>q^{c}\left(z, x^{\prime}\right)$. Soit alors $\mu$ vérifiant $F_{z}\left(x^{\prime}\right)>\mu>q^{c}\left(z, x^{\prime}\right)$. En utilisant la définition de $q^{c}\left(z, x^{\prime}\right)$, on obtient :

$$
\operatorname{Inf}\left\{\left\langle z-x, x^{\prime}\right\rangle: f(x) \leqq \mu\right\} \leqq 0<\mu
$$

Par conséquent il existe $u$ appartenant à $X$ pour lequel $f(u) \leqq \mu$ et $\left\langle z-u, x^{\prime}\right\rangle \leqq \mu$, autrement dit $F_{z}\left(x^{\prime}\right) \leqq f(u) \vee\left\langle z-u, x^{\prime}\right\rangle \leqq \mu<F_{z}\left(x^{\prime}\right)$, ce qui est absurde et donc $F_{z}\left(x^{\prime}\right) \leqq q^{c}\left(z, x^{\prime}\right)$. Soit $x^{\prime} \in Q f(z)$. Par définition, on a : $f(z)=F_{z}\left(x^{\prime}\right)$. Comme $F_{z}\left(x^{\prime}\right) \leqq q^{c}\left(z, x^{\prime}\right)$, on déduit que: $f(z) \leqq q^{c}\left(z, x^{\prime}\right)$. Or $q^{c}\left(z, x^{\prime}\right) \leqq f(z)$, d'où $q^{c}\left(z, x^{\prime}\right)=f(z)$ et donc $x^{\prime} \in T f(z)$. @ 


\section{OPTIMISATION QUASI-CONVEXE}

A $f$ appartenant à $P$, on associe le problème primal $(P): \alpha=\operatorname{Inf} f$.

Désignons par $U$ et $U^{\prime}$ deux espaces vectoriels topologiques localement convexes en dualité par la forme bilinéaire notée $($,$) . U$ est appelé espace de perturbations pour le problème $(P)$.

Soit $\psi$ la fonction de perturbation de $X \times U \rightarrow[0,+\infty]$ telle que :

$$
\psi(x, 0)=f(x) \text { pour tout } x \text { appartenant à } X
$$

Pour la perturbation $u$ de $U$, on considère le problème perturbé $\left(P_{u}\right)$ :

$$
k(u)=\operatorname{Inf}\{\psi(x, u): x \in X\}
$$

Remarquons que le problème $(P)$ correspond à la valeur $u=0$ et donc $\alpha=k(0) . k^{E}$ étant la plus grande minorante qcx et sci de $k$, on déduit que $k^{E}(0) \leqq k(0)=\alpha$. Cette inégalité nous amène à considérer le problème dual $(Q) \operatorname{de}(P)$ :

$$
\beta=\operatorname{Sup}\left\{K_{0}\left(u^{\prime}\right): u^{\prime} \in U^{\prime}\right\}
$$

Son expression est :

$$
\beta=\operatorname{Sup}\left\{\operatorname{Inf}\left\{\left[\operatorname{Inf}\{\psi(x, u): x \in X\} \vee\left(-u, u^{\prime}\right)\right]: u \in U\right\} u^{\prime} \in U^{\prime}\right\}
$$

On a toujours $\beta \leqq \alpha$; cependant on a l'égalité si $k$ est qcx et sci en 0 .

Désignons par $\mathrm{S}$ l'ensemble des solutions du problème $(Q)$. On a le résultat suivant.

THÉORÈME 7 : Si l'ensemble $Q f(0)$ est non vide, alors

$$
\beta=\alpha \Leftrightarrow Q k(0)=S
$$

Preuve: Il suffit de remarquer que:

$S=\left\{u^{\prime} \in U^{\prime}: K_{0}\left(u^{\prime}\right)=\beta=k^{E}(0)\right\} \quad$ et $\quad Q f(0)=\left\{u^{\prime} \in U^{\prime}: K_{0}\left(u^{\prime}\right)=\alpha\right\}$.

Exemple: la «surrogate duality».

Considérons le problème suivant. Soient $X=R,($, ) le produit scalaire usuel, $f \in P$ et $g \in R^{X}$.

Posons $(P): \alpha=\operatorname{Inf}\{f(x) ; x \in C\}$ où $C=T(g, 0)$.

On se ramène au cas précédent en posant $\mathbf{f}(x)=f(x)$ si $x \in C$ et $+\infty$ sinon.

Posons $U=R$ et soit le problème perturbé: $k(u)=\operatorname{Inf}\left\{f(x): x \in C_{u}\right\}$ où à $u \in U$, on associe l'ensemble $C_{u}=T(g, u)$. 
Pour être conforme avec nos notations précédentes, on prendra comme fonction de perturbation $\psi(x, u)=f(x)$ si $x \in C_{u}$ et $+\infty$ sinon. On a :

$$
\begin{aligned}
\psi_{(z, t)}\left(0, u^{\prime}\right)=\operatorname{Inf}\left\{\psi(x, u) \vee\left(t-u, u^{\prime}\right): x, u \in \mathrm{R}\right\} & \\
& =\operatorname{Inf}\left\{f(x) \vee\left(t-u, u^{\prime}\right): x \in C_{u}, u \in R\right\}
\end{aligned}
$$

et

$$
\begin{aligned}
K_{t}\left(u^{\prime}\right)=\operatorname{Inf}\left\{k(u) \vee\left(t-u, u^{\prime}\right): u\right. & \in U\} \\
& =\operatorname{Inf}\left\{\operatorname{Inf}\left\{f(x) ; x \in C_{u}\right\} \vee\left(t-u, u^{\prime}\right): u \in R\right\} .
\end{aligned}
$$

On remarque alors que :

$$
\Psi_{(z, t)}\left(0, u^{\prime}\right)=\mathrm{K}_{t}\left(u^{\prime}\right)
$$

et

$$
\beta=\operatorname{Sup}\left\{\operatorname{Inf}\left\{f(x) \vee\left(-u, u^{\prime}\right): g(x) \leqq u, u \in R\right\} u^{\prime} \in R\right\} .
$$

Posons $d=\operatorname{Inf}\left\{f(x) \vee\left(-u, u^{\prime}\right): g(x) \leqq u, u \in R\right\}$.

(i) $u^{\prime} \geqq 0 \Rightarrow \operatorname{Inf}\left\{f(x) \vee\left(-u, u^{\prime}\right): g(x) \leqq u, 0 \leqq u\right\}$

$$
=\operatorname{Inf}\{f(x): g(x) \leqq u, 0 \leqq u\}=\operatorname{Inf} f \Rightarrow d=\operatorname{Inf} f .
$$

(ii) $u^{\prime}<0 \Rightarrow \operatorname{Inf}\left\{f(x) \vee\left(-u, u^{\prime}\right): g(x) \leqq u, u \leqq 0\right\}$

$$
\begin{gathered}
=\operatorname{Inf}\{f(x): g(x) \leqq 0\}=\alpha \\
\geqq \operatorname{Inf}\left\{\operatorname{Max}\left[f(x),-u \cdot u^{\prime}\right]: g(x) \leqq u, 0 \leqq u\right\} \\
=\operatorname{Inf}\left\{\operatorname{Max}\left[f(x),-g(x) \cdot u^{\prime}\right]: x \in X\right\} \\
\Rightarrow d=\operatorname{Inf}\left\{\operatorname{Max}\left[f(x) \vee\left(-g(x), u^{\prime}\right): x \in X\right\} .\right.
\end{gathered}
$$

En résumé, le problème s'écrit:

$$
\beta=\operatorname{Sup}\left\{d: u^{\prime} \in U\right\}=\operatorname{Sup}\left\{\operatorname{Inf}\left\{f(x) \vee\left(-g(x), u^{\prime}\right): x \in X\right\} u^{\prime} \leqq 0\right\} .
$$

\section{CONCLUSION}

On a traité le cas où $f$ est positive. Si $f$ est négative, on utilise la supconvolution quasi-convexe pour définir la polaire de $f$ en un point $z$. On notera :

$n^{f}\left(z, x^{\prime}\right)=\operatorname{Sup}\left\{\operatorname{Min}\left[f(x),\left\langle z-x, x^{\prime}\right\rangle\right]: x \in X\right\}$ pour tout $x^{\prime}$ appartenant à $X^{\prime}$ 
Dans ce cas, $n^{f}(z, 0)=\operatorname{Sup} f$.

Cependant une question demeure en suspens: peut-on prolonger notre construction à des fonctionnelles à valeurs dans $[-\infty,+\infty]$ ?

Note de l'auteur. - Je tiens à remercier Réda Rheffouli pour ses remarques fructueuses et les arbitres dont j'ai beaucoup apprécié les critiques constructives.

\section{BIBLIOGRAPHIE}

1. M. Atteia et A. Elqortobl, Quasi-Convex Duality Lectures Notes in Control and Inform. Sci., 1980, 30, p. 3-8.

2. N. Bourbaki, Espaces Vectoriels topologiques, Hermann, Paris,

3. J. P. Crouzerx, Contributions à l'étude des fonctions quasiconvexes, Thèse de Doctorat d'État, série $\mathrm{E}, \mathrm{n}^{\circ}$ d'ordre 250, 1977. Université de Clermont-Ferrand (France).

4. M. J. Greenberg et W. P. Pierskalla, Quasi-Conjugate Functions and Surrogate Duality, Cahiers Centre Études Rech. Opér., 1973, 15, p. 437-448.

5. P. J. Laurent, Approximation et Optimisation, Hermann, Paris, 1972.

6. J. E. Martinez-Legaz, Quasiconvex Duality Theory by Generalized Conjugation Methods, Optimization, 1988, 19, p. 603-652.

7. J. J. Moreau, Inf-convolution, sous-additivité, convexité des fonctions numériques, J. Math. Pures Appl., 1970, 49, p. 109-154.

8. U. Passy et E. Z. Prisman, Conjugacy in quasiconvex programming, Math. Programming, 1984, 30, p. 121-146.

9. J. P. Penot et M. Volle, On Quasi-convex Duality, Math. Oper. Res., 1990, 15, p. $597-625$.

10. R. T. Rockafellar, Convex Analysis, Princeton, 1970.

11. I. Singer, Conjugate Functionals and Level Sets, Nonlinear Anal. Theory, Methods Appl., 1984, 8, p. 313-320.

12. M. Volle, Conjugaison par tranches, Ann. Mat. Pura Appl. (IV), 1985, 139, p. 279-312. 